Research Paper

\title{
Risk Factors for Discontinuation of S-1 Adjuvant Chemotherapy for Gastric Cancer
}

\author{
Hitoshi Kawazoe ${ }^{1,}$, , Maya Shimasaki ${ }^{2,}$, Masaki Ueno ${ }^{1}$, Satomi Sumikawa1, Shingo Takatori2, Hiroyuki \\ Namba $^{2}$, Motohira Yoshida ${ }^{3}$, Koichi Sato ${ }^{3}$, Yoh Kojima ${ }^{3}$, Yuji Watanabe ${ }^{3}$, Toshihide Moriguchi ${ }^{1}$, Akihiro \\ Tanaka $^{1 凶}$, and Hiroaki Araki ${ }^{1}$ \\ 1. Division of Pharmacy, Ehime University Hospital, Shitsukawa, Toon, Ehime 791-0295, Japan; \\ 2. Department of Clinical Pharmacy, College of Pharmaceutical Sciences, Matsuyama University, Bunkyou, Matsuyama, Ehime 790-8578, Japan; \\ 3. Division of Gastrointestinal Surgery and Surgical Oncology, Department of Surgery, Ehime University Graduate School of Medicine, Shitsukawa, \\ Toon, Ehime 791-0295, Japan. \\ * Hitoshi Kawazoe and Maya Shimasaki equally contributed to this work.
}

$\triangle$ Corresponding author: Akihiro Tanaka, Division of Pharmacy, Ehime University Hospital, Shitsukawa, Toon, Ehime 791-0295, Japan. Phone: +81-89-960-5738; Fax: +81-89-960-5745; E-mail: akiki@m.ehime-u.ac.jp

() 2015 Ivyspring International Publisher. Reproduction is permitted for personal, noncommercial use, provided that the article is in whole, unmodified, and properly cited. See http://ivyspring.com/terms for terms and conditions.

Received: 2014.11.27; Accepted: 2015.01.03; Published: 2015.03.18

\begin{abstract}
Purpose: The aim of this study was to clarify the risk factors for discontinuing tegafur/gimeracil/oteracil potassium (S-1) adjuvant chemotherapy following gastrectomy in patients with gastric cancer.

Methods: We retrospectively investigated patients with curatively-resected gastric cancer who received S- 1 adjuvant chemotherapy. S- 1 was administered orally at $80-120 \mathrm{mg} /$ day, depending on body surface area, on days 1-28 every 6 weeks for 1 year. The dose and treatment schedule were modified at the clinicians' discretion, according to toxicity.

Results: Seventy-one patients were included in the study, 26 of whom discontinued S-1 therapy. The relapse-free survival rates in the S-1-completed and S-1-discontinuation groups at 5 years post-surgery were $88.1 \%$ and $55.8 \%$, respectively. The overall survival rates in the $\mathrm{S}-1$-completed and S-1-discontinuation groups at 5 years post-surgery were $89.4 \%$ and $59.8 \%$, respectively. The hazard ratios for relapse and death were significantly lower in the $\mathrm{S}$-1-completed group compared with those in the S-1-discontinuation group ( $0.18 ; p<0.001$ and $0.19 ; p=0.002$, respectively). Multivariate logistic regression analysis revealed that $\mathrm{S}-1$ discontinuation was significantly associated with an initial overdose of S-1, having stage I cancer, creatinine clearance $<66 \mathrm{~mL} / \mathrm{min}$, and a side effect of nausea.

Conclusions: These results suggest that assessing renal function to avoid initial overdose of S-1, together with the early management of side effects, may support the continuation of S-1 adjuvant chemotherapy in patients with gastric cancer.
\end{abstract}

Key words: Adjuvant chemotherapy; Tegafur/gimeracil/oteracil potassium (S-1); Gastric cancer; Discontinuation; Risk factor.

\section{Introduction}

Gastric cancer is the second leading cause of cancer-related death, with the highest mortalities in East Asia, including Japan, Korea, and China. ${ }^{1}$ D2 gastrectomy is commonly regarded as the standard surgical procedure for advanced gastric cancer in East Asia. However, over $40 \%$ of patients experience cancer recurrence after gastrectomy. ${ }^{2} \mathrm{~S}-1$ is an oral fluoropyrimidine comprising tegafur, gimeracil 
(CDHP), and oteracil potassium, and is used as a standard postoperative adjuvant chemotherapy agent in patients with stage II or III gastric cancer in Japan. ${ }^{3}$ The phase III Adjuvant Chemotherapy Trial of S-1 for Gastric Cancer (ACTS-GC) found that $34.2 \%$ of patients had discontinued S- 1 at 1 year post-surgery. ${ }^{4}$ This low completion rate of S-1 treatment remains a clinically-unresolved issue, though information on the reasons for discontinuation is sparse. In addition, a post-hoc analysis of the ACTS-GC trial data, which have not been published in a journal, showed that patients who completed the planned 1-year S-1 treatment survived longer than patients who discontinued S-1. However, data on compliance, dose reduction, and treatment schedule modifications during S-1 adjuvant chemotherapy in gastric cancer patients in clinical practice are lacking. Adjuvant chemotherapy aims to increase the probability of a cancer cure, and increasing the currently low completion rate of S-1 adjuvant chemotherapy is thus an important issue. Identification of the risk factors associated with discontinuation of S-1 adjuvant chemotherapy would allow clinicians and community pharmacists to support patients with those risk factors. We therefore retrospectively investigated the risk factors for discontinuation of S-1 adjuvant chemotherapy in patients with gastric cancer.

\section{Materials and methods}

\section{Patients and study design}

This retrospective observational study was carried out at Ehime University Hospital using electronic medical record data. We extracted the necessary clinical information on patient demographics, compliance, treatment outcomes, and toxicities. Between August 2006 and February 2014, we investigated patients with curatively-resected gastric cancer who received S-1 adjuvant chemotherapy. S-1 was administered at $80-120 \mathrm{mg} /$ day, depending on body surface area (BSA), on days 1-28 every 6 weeks (in principle) for 1 year, in the absence of recurrence, unacceptable side effects, or patient refusal. Patients with a BSA $<1.25 \mathrm{~m}^{2}$ received $80 \mathrm{mg} /$ day; patients with a BSA of $1.25-1.5 \mathrm{~m}^{2}$ received $100 \mathrm{mg} /$ day; and patients with a BSA $>1.5 \mathrm{~m}^{2}$ received $120 \mathrm{mg} /$ day. The dose or treatment schedule of S-1 was modified at the clinicians' discretion, according to the toxicity profiles. The clinicopathologic findings were determined in accordance with the Japanese classification of gastric carcinoma. ${ }^{5}$ Consenting patients with stages I-IV (M0) gastric cancer were candidates for adjuvant chemotherapy. Creatinine clearance (Ccr) was calculated using the Cockcroft-Gault formula by adding 0.2 $\mathrm{mg} / \mathrm{dL}$ to the serum creatinine level measured by the enzymatic peroxidase-antiperoxidase method. ${ }^{6}$ According to the pharmaceutical company's guide, we categorized each initial S-1 dose for patients with a Ccr $<60 \mathrm{~mL} / \mathrm{min}$ as an underdose, standard dose, or overdose. For example, patients with a BSA $>1.5 \mathrm{~m}^{2}$ received $120 \mathrm{mg} /$ day standard dose, but patients with a low Ccr $<60 \mathrm{~mL} / \mathrm{min}$ received a standard dose of $100 \mathrm{mg} /$ day. A total of 88 patients were enrolled, but 17 were subsequently excluded because of hospital transfer $(n=10)$, ongoing treatment $(n=3)$, or a Ccr of $<30 \mathrm{~mL} / \mathrm{min}(n=4)$. The 71 remaining patients were assessed and relapse-free survival (RFS) and overall survival (OS) were compared between patients who completed the schedule S-1 treatment (S-1-completed group) and those who discontinued treatment (S-1-discontinuation group). A further analysis was performed to assess the risk factors associated with S-1 discontinuation after the exclusion of seven patients who discontinued S- 1 because of relapse $(n=64)$.

The study protocol was approved by the ethics committee of Ehime University Hospital (approval number: 1402005) and was conducted in accordance with the ethical principles of the Japanese ethics guidelines for epidemiological studies.

\section{Statistical analysis}

RFS was defined as the interval from the date of surgery to the date when relapse was confirmed by computed tomography or positron emission tomography-computed tomography, death from any cause, or last follow-up, whichever came first. OS was defined as the interval from the date of surgery to the date of death from any cause or last follow-up. RFS and OS were compared between the S-1-completed and S-1-discontinuation groups using the Kaplan-Meier method. Hazard ratios (HRs) for relapse and death were compared between the completed and discontinuation groups using log-rank tests. Patient characteristics and side effects were compared using Student's $t$, Welch's $t$, and $\chi^{2}$ tests, as appropriate. Multivariate logistic regression analysis was used to evaluate the risk factors associated with S-1 discontinuation. Possible explanatory variables with $p<0.05$ in univariate analysis were included in the multivariate model. Cut-off values for continuous variables such as serum albumin and Ccr were calculated using receiver operating characteristic (ROC) curves and categorized as categorical variables. The relative dose intensity (RDI) of S-1 was defined as the actual dose divided by the planned dose during the period of S-1 treatment. All analyses were performed using JMP 8.0 (SAS Institute, Tokyo, Japan). All $p$ values were two-tailed, and $p<0.05$ was considered significant. 


\section{Results}

The CONSORT diagram is shown in Fig. 1. Seventy-one patients were included in the study, 26 patients $(36.6 \%)$ of whom discontinued S-1 therapy. There were no significant differences between the S-1-completion and S-1-discontinuation groups in terms of baseline characteristics, except for ages, serum albumin levels, and Cor levels (Table 1). The overall median follow-up time was 26.4 months. There were 15 relapse events and 12 deaths in both groups combined.

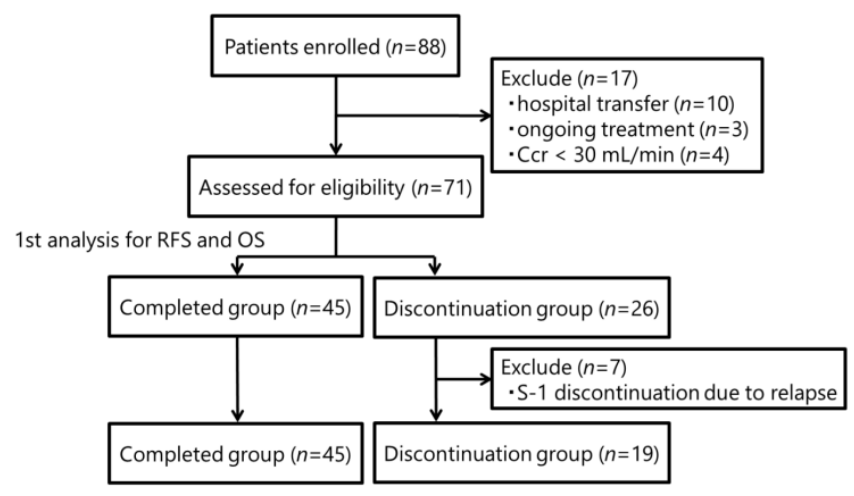

2nd analysis for the risk factors of S-1 discontinuation

Figure 1. CONSORT diagram. Ccr: creatinine clearance; RFS: relapse-free survival; OS: overall survival

Table 1. Baseline characteristics of the patients.

\begin{tabular}{|c|c|c|c|c|}
\hline & & $\begin{array}{l}\text { Completed } \\
\text { group }(n=45)\end{array}$ & $\begin{array}{l}\text { Discontinuation } \\
\text { group }(n=26)\end{array}$ & $\begin{array}{l}p \text { val- } \\
\text { ue }^{\mathrm{b}}\end{array}$ \\
\hline \multirow[t]{2}{*}{ Sex, $n(\%)$} & male & $29(64.4)$ & $18(69.2)$ & 0.681 \\
\hline & female & $16(35.6)$ & $8(30.8)$ & \\
\hline \multicolumn{2}{|l|}{ Age (years)a } & $67[25-87]$ & $72[46-89]$ & 0.048 \\
\hline \multicolumn{2}{|c|}{ Body mass index $\left(\mathrm{kg} / \mathrm{m}^{2}\right)^{a}$} & 21.5 [14.7-31.2] & 21.5 [16.9-26.9] & 0.798 \\
\hline \multicolumn{2}{|l|}{$\operatorname{BSA}\left(\mathrm{m}^{2}\right)^{\mathrm{a}}$} & $1.55[1.28-2.04]$ & 1.59 [1.34-1.83] & 0.662 \\
\hline \multicolumn{2}{|l|}{ Serum albumin $(\mathrm{g} / \mathrm{dL})^{\mathrm{a}}$} & $3.8[3.0-4.5]$ & $3.6[2.4-4.3]$ & 0.011 \\
\hline \multicolumn{2}{|l|}{$\mathrm{Ccr}(\mathrm{mL} / \mathrm{min})^{\mathrm{a}}$} & $57.1[32.7-118.4]$ & 51.6 [35.0-95.2] & 0.036 \\
\hline \multirow{4}{*}{$\begin{array}{l}\text { Surgical procedure, } n \\
(\%)\end{array}$} & LATG & $2(4.4)$ & $1(3.8)$ & 0.825 \\
\hline & LADG & $18(40.0)$ & $12(46.2)$ & \\
\hline & TG & $10(22.2)$ & $7(26.9)$ & \\
\hline & DG & $15(33.3)$ & $6(23.1)$ & \\
\hline \multirow[t]{4}{*}{ Stage, $n(\%)$} & I & $8(17.8)$ & $8(30.8)$ & 0.435 \\
\hline & II & $14(31.1)$ & $7(26.9)$ & \\
\hline & III & $15(33.3)$ & $5(19.2)$ & \\
\hline & IV & $8(17.8)$ & $6(23.1)$ & \\
\hline
\end{tabular}

BSA: body surface area; Ccr: creatinine clearance; LATG: laparoscopic-assisted total gastrectomy; LADG: laparoscopic-assisted distal gastrectomy; TG: total gastrectomy; DG: distal gastrectomy. a Values shown as median [range]. b Characteristics were compared between the S-1-completed and discontinuation groups using Student's $t$-tests for body mass index, Welch's $t$-tests for age, body surface area, serum albumin level, and Ccr, and $x^{2}$ tests for sex, surgical procedure, and cancer stage.

The RFS rates at 5 years post-surgery in the S-1-completed and S-1-discontinuation groups were $88.1 \%$ and $55.8 \%$, respectively (Fig. 2A). The HR for relapse in the S-1-completed group compared with that in the discontinuation group was 0.18 (95\% confidence interval [CI] 0.04-0.38; $p<0.001$ ), indicating that completing S-1 treatment reduced the risk of relapse by $82 \%$. The OS rates at 5 years post-surgery in the S-1-completed and S-1-discontinuation groups were $89.4 \%$ and $59.8 \%$, respectively (Fig. $2 \mathrm{~B}$ ). The HR for death in the S-1-completed group compared with that in the discontinuation group was 0.19 (95\% CI $0.04-0.49 ; p=0.002)$. Completion of S-1 treatment thus reduced the risk of death by $81 \%$.

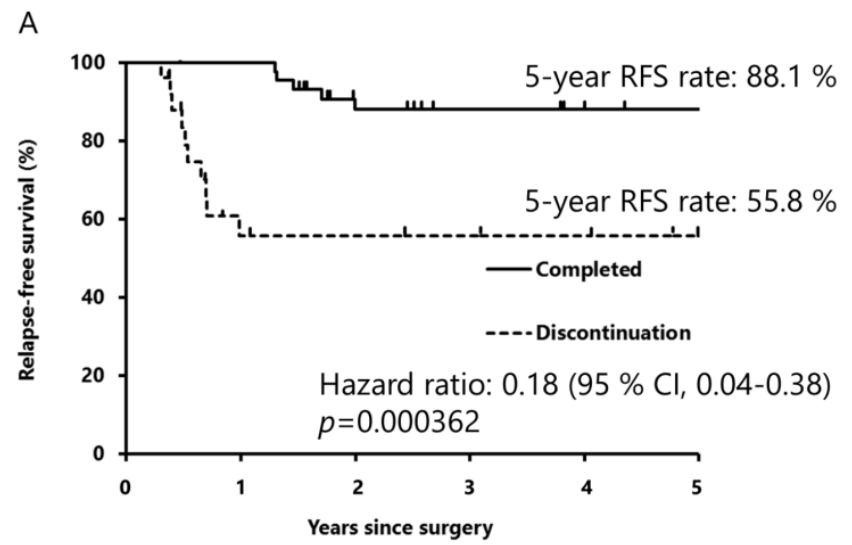

B

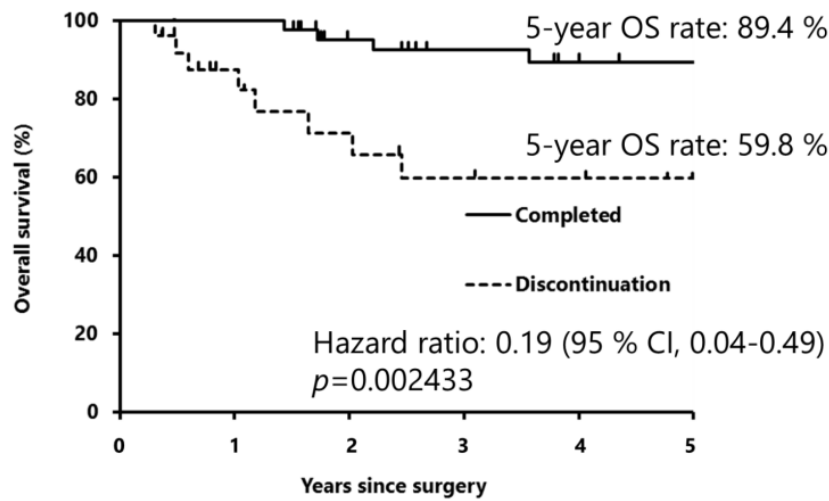

Figure 2. Kaplan-Meier curves for relapse-free survival (A) and overall survival (B). The differences in relapse-free and overall survival between the S-1-completed and S-1-discontinuation groups were calculated using the Kaplan-Meier method, and the hazard ratios for relapse and death in the $\mathrm{S}-1$-completed and discontinuation groups were analyzed by log-rank tests. RFS: relapse-free survival; $\mathrm{Cl}$ : confidence interval; OS: overall survival

We subsequently evaluated the risk factors associated with S-1 discontinuation, excluding seven patients who discontinued S-1 because of relapse. Digestive symptoms and hematological toxicity of any grade were frequent side effects of S-1 (Table 2). The reasons for S-1 discontinuation were side effects in 16 patients $(84.2 \%)$ and patient refusal in three patients $(15.8 \%)$ (Table 3$)$. Dose reduction was required during treatment in 20 patients $(44.4 \%)$ in the S-1-completion group and five patients $(26.3 \%)$ in the S-1-discontinuation group. The treatment schedule was modified during treatment in 42 patients (93.3\%) in the S-1-completion group and eight patients $(42.1 \%)$ in the discontinuation group. 
Univariate analysis revealed that S-1 discontinuation was not significantly associated with age, S-1 modification/dose reduction during treatment, surgical procedure, complications including diabetes mellitus or hypertension, prescription of prophylactic antidiarrheal, laxative, probiotic, and antiemetic agents, prescription of therapeutic antidiarrheal, probiotic, and antiemetic agents, side effects including vomiting, diarrhea, stomatitis, anorexia, dysgeusia,

Table 2. Side effects induced by S-1 $(n=64)$.

\begin{tabular}{llll}
\hline & $\begin{array}{l}\text { Completed } \\
\text { group }(n=45)\end{array}$ & $\begin{array}{l}\text { Discontinuation } \\
\text { group }(n=19)\end{array}$ & $\begin{array}{l}p \\
\text { value }^{\mathrm{a}}\end{array}$ \\
\hline $\begin{array}{l}\text { Non-hematological toxicity, } n \\
(\%)\end{array}$ & Any grade & Any grade & \\
Nausea & $6(13.3)$ & $8(42.1)$ & 0.027 \\
Vomiting & $5(11.1)$ & $4(21.1)$ & 0.515 \\
Diarrhea & $26(57.8)$ & $8(42.1)$ & 0.251 \\
Stomatitis & $12(26.7)$ & $3(15.8)$ & 0.538 \\
Anorexia & $21(46.7)$ & $7(36.8)$ & 0.469 \\
Dysgeusia & $6(13.3)$ & $4(21.1)$ & 0.689 \\
Pigmentation & $8(17.8)$ & $3(15.8)$ & 0.865 \\
Skin rash & $7(15.6)$ & $4(21.1)$ & 0.865 \\
Lacrimation & $13(28.9)$ & $3(15.8)$ & 0.430 \\
Others & $33(73.3)$ & $10(52.6)$ & 0.107 \\
Hematological toxicity, $n(\%)$ & & & \\
Leucopenia & $32(71.1)$ & $7(36.8)$ & 0.010 \\
Neutropenia & $31(68.9)$ & $6(31.6)$ & 0.006 \\
Anemia & $43(95.6)$ & $16(84.2)$ & 0.300 \\
Thrombocytopenia & $17(37.8)$ & $5(26.3)$ & 0.378
\end{tabular}

aSide effects were compared between the S-1-completed and discontinuation groups using $\chi^{2}$ tests. pigmentation, skin rash, lacrimation, anemia, thrombocytopenia, and others. Variables that showed a significant difference in univariate analysis were included in a multivariate model. Multivariate logistic regression analysis revealed that S-1 discontinuation was significantly associated with an initial overdose of S-1, having stage I cancer, $\mathrm{Ccr}<66 \mathrm{~mL} / \mathrm{min}$, and a side effect of nausea (Fig. 3).

Table 3. Reasons for S-1 discontinuation $(n=19)$.

\begin{tabular}{ll}
\hline Side effects, $n(\%)$ & $16(84.2)$ \\
\hline Nausea & 3 \\
Anorexia & 2 \\
Diarrhea & 2 \\
Thrombocytopenia & 2 \\
Hand-foot syndrome & 1 \\
Ileus & 1 \\
Abdominal pain & 1 \\
Infection & 1 \\
Dysgeusia & 1 \\
Lacrimation & 1 \\
Interstitial pneumonia & 1 \\
Fatigue & 1 \\
Unknown & 3 \\
Patient refusal & 3 (15.8) \\
\hline The numbers do not total 19 because some patients cited multiple reasons for \\
discontinuation.
\end{tabular}

\begin{tabular}{|c|c|c|c|c|c|c|c|}
\hline \multicolumn{2}{|l|}{ Risk factors } & \multirow{2}{*}{$\begin{array}{c}\text { Case / Control } \\
3 / 10\end{array}$} & \multirow[t]{2}{*}{ Less } & \multirow[t]{2}{*}{ More } & Adjusted OR & \multirow{2}{*}{$\begin{array}{r}95 \% \text { CI } \\
(0.439-259)\end{array}$} & \multirow{2}{*}{$\frac{p \text { value }}{0.163}$} \\
\hline Initial S-1 dose & underdose & & & & 7.776 & & \\
\hline & standard & $7 / 26$ & & & 1.000 & & \\
\hline & overdose & $9 / 9$ & & & 14.483 & $(1.038-600)$ & 0.047 \\
\hline \multirow[t]{2}{*}{ Schedule change } & Yes & $8 / 42$ & & & 0.031 & $(0.001-0.307)$ & 0.002 \\
\hline & No & $11 / 3$ & & & 1.000 & & \\
\hline \multirow[t]{2}{*}{ Stage } & I & $8 / 8$ & & & 17.873 & $(1.650-441)$ & 0.016 \\
\hline & others & $11 / 37$ & & & 1.000 & & \\
\hline \multirow[t]{2}{*}{ Serum albumin $(g / d L)$} & $<3.3$ & $8 / 5$ & & & 4.786 & $(0.436-64.484)$ & 0.196 \\
\hline & $\geq 3.3$ & $11 / 40$ & & & 1.000 & & \\
\hline \multirow[t]{2}{*}{$\operatorname{Ccr}(\mathrm{mL} / \mathrm{min})$} & $<66$ & $16 / 27$ & & & 25.829 & $(1.162-3040)$ & 0.039 \\
\hline & $\geq 66$ & $3 / 18$ & & & 1.000 & & \\
\hline \multirow[t]{2}{*}{ Laxative prescription } & Yes & $1 / 11$ & & 4 & 0.064 & $(0.000-2.175)$ & 0.145 \\
\hline & No & $18 / 34$ & & & 1.000 & & \\
\hline \multirow[t]{2}{*}{ Nausea } & Yes & $8 / 6$ & & & 25.684 & $(2.079-1001)$ & 0.010 \\
\hline & No & $11 / 39$ & & & 1.000 & & \\
\hline \multirow[t]{3}{*}{ Neutropenia } & Yes & $6 / 31$ & & & 0.163 & (0.014-1.351) & 0.093 \\
\hline & No & $13 / 14$ & & & 1.000 & & \\
\hline & & & 0.1 & 10 & 100 & & \\
\hline & & & & & & & \\
\hline
\end{tabular}

Figure 3. Forest plot of risk factors associated with S-1 discontinuation. Multivariate logistic regression analysis was used to evaluate the risk factors associated with S-1 discontinuation. OR: odds ratio; Cl: confidence interval; Ccr: creatinine clearance 


\section{Discussion}

There is currently little information on the optimal management of S-1 adjuvant chemotherapy in patients with gastric cancer in the clinical practice setting. The present study showed that the S-1 discontinuation rate was $36.6 \%$, which was similar to the dropout rate identified in the previous ACTS-GC trial. ${ }^{4}$ In addition, patients who completed S-1 chemotherapy benefited from lower relapse and death rates than those who discontinued S-1 chemotherapy, suggesting that it was a very important evidence to maintain a continuous administration of S-1 after gastrectomy in patients with gastric cancer. Furthermore, multivariate logistic regression analysis demonstrated the first report that S-1 discontinuation was significantly associated with an initial overdose of S-1, having stage I cancer, a Ccr $<66 \mathrm{~mL} / \mathrm{min}$, and a side effect of nausea.

Recent studies reported that the dose of S-1 should take into consideration the renal function of each patient, with the aim of enabling patients to complete 1 year of S-1 treatment.2,7,8 S-1 includes CDHP, an inhibitor of dihydropyrimidine dehydrogenase (DPD), which is the rate-limiting enzyme for the degradation of fluorouracil. ${ }^{9} \mathrm{CDHP}$ clearance depends on renal function, resulting in high blood concentrations of fluorouracil because of reduced DPD activity. This may lead to severe and/or frequent S-1-induced side effects in patients with low Ccr levels. Yamanaka et al. reported that baseline renal function was a significant risk factor for severe neutropenia caused by S-1 chemotherapy in advanced gastric cancer. ${ }^{10}$ Iwasa et al. reported that patients with low Ccr levels tended to require S-1 dose reduction. ${ }^{7}$ A recent 6-month study found that $\mathrm{Ccr}<60 \mathrm{~mL} / \mathrm{min}$ was a significant risk factor for discontinuation of S-1 adjuvant chemotherapy. ${ }^{8}$ Our results showed that S-1 discontinuation was significantly associated with an initial overdose of S-1 and Ccr $<66 \mathrm{~mL} / \mathrm{min}$. We excluded four patients with $\mathrm{Ccr}<30 \mathrm{~mL} / \mathrm{min}$ because the pharmaceutical company's guidance cites $\mathrm{Ccr}<30$ $\mathrm{mL} / \mathrm{min}$ as a contraindication to S- 1 therapy. The appropriate initial dose of S-1 of $80-120 \mathrm{mg} /$ day should thus take into consideration not only BSA, but also pretreatment Cor level, paying particular attention to patients with $\mathrm{Ccr}<66 \mathrm{~mL} / \mathrm{min}$. Clinicians and community pharmacists can provide support for patients with the above risk factors.

In the present study, the dose of S-1 was reduced in $44.4 \%$ of patients in the S-1-completed group, which was comparable to that in the ACTS-GC trial $(46.5 \%)$. In addition, $93.3 \%$ of patients in the S-1-completion group changed their treatment schedule. Iwasa et al. reported that the planned 1 year
S-1 adjuvant therapy for stage II or III gastric cancer could be completed in most patients by modifying the dose and/or treatment schedule. ${ }^{7}$ Kim et al., however, reported that a decreased RDI was related to poor DFS in patients with stage II-IV gastric cancer who underwent curative surgery and received S-1 adjuvant chemotherapy. ${ }^{2}$ In this study the HRs for relapse and death in the S-1-completion group were significantly lower ( 0.18 and 0.19 , respectively) than those in the discontinuation group. The average RDI in the S-1-completion group was $94.4 \%$ with a $95 \%$ CI of $53.6-127.1 \%$. However, we were unable to calculate the optimal RDI cut-off value using an ROC curve because of the small sample size. Previous studies found that persistent gastrointestinal toxicities, even of low grade, were the major reason for patient refusal to continue S-1 treatment. 4,7 In the present study, S-1-induced digestive symptoms were relatively common side effects, as seen in the ACTS-GC trial. Our results showed that having stage I cancer and a side effect of nausea were risk factors for discontinuing S- 1 adjuvant chemotherapy. It is possible that S-1 adjuvant chemotherapy might be associated with both less benefit and more side effects in patients with stage I cancer, resulting in patient refusal to continue treatment. However, a dose-reduction of one level should be considered in patients with uncomfortable digestive symptoms: from $120 \mathrm{mg}$ to $100 \mathrm{mg} /$ day, from $100 \mathrm{mg}$ to $80 \mathrm{mg} /$ day, or from $80 \mathrm{mg}$ to 60 $\mathrm{mg} /$ day. Maximum effort should be made to maintain dose intensity, in light of these results.

The present study had several limitations. First, it was a retrospective, single-institution study with a small sample size. Second, we assessed consenting patients with stages I-IV disease (M0) who were candidates for adjuvant chemotherapy, whereas patients with stage II or III gastric cancer were eligible for the ACTS-GC trial. ${ }^{4}$ There may thus have been some bias in our results. Large-scale, multicenter studies are needed to confirm the results of this study.

This study is the first to clarify the risk factors for discontinuation of S-1 adjuvant chemotherapy 1 year post-surgery in patients with gastric cancer in a clinical setting, using multivariate logistic regression analysis. The results suggest that patient renal function should be assessed to avoid initial S-1 overdose. Early management of S-1-induced side effects, especially nausea, may also support the continuation of S-1 adjuvant chemotherapy in patients with gastric cancer.

\section{Acknowledgements}

We thank Ms. Autumn Albers for editing assistance in the preparation of this manuscript. 


\section{Competing Interests}

The authors have declared that no competing interest exists.

\section{References}

1. Sasako M, Sakuramoto S, Katai H, et al. Five-year outcomes of a randomized phase III trial comparing adjuvant chemotherapy with S-1 versus surgery alone in stage II or III gastric cancer. J Clin Oncol. 2011; 29: 4387-93.

2. Kim SJ, Kim YJ, Kim JH, et al. Safety, compliance, and predictive parameters for dosage modification in adjuvant S-1 chemotherapy for gastric cancer. Cancer Sci. 2013; 104: 116-23.

3. Sano T, Aiko T. New Japanese classifications and treatment guidelines for gastric cancer: revision concepts and major revised points. Gastric Cancer. 2011; 14: 97-100.

4. Sakuramoto S, Sasako M, Yamaguchi T, et al. Adjuvant chemotherapy for gastric cancer with S-1, an oral fluoropyrimidine. N Engl J Med. 2007; 357: 1810-20.

5. Japanese Gastric Cancer Association. Japanese classification of gastric carcinoma-2nd English edition. Gastric Cancer. 1998; 1: 10-24.

6. Shimokata T, Ando Y, Yasuda Y, et al. Prospective evaluation of pharmacokinetically guided dosing of carboplatin in Japanese patients with cancer. Cancer Sci. 2010; 101: 2601-5.

7. Iwasa S, Yamada Y, Fukagawa T, et al. Management of adjuvant S-1 therapy after curative resection of gastric cancer: dose reduction and treatment schedule modification. Gastric Cancer. 2011; 14: 28-34.

8. Aoyama T, Yoshikawa T, Hayashi T, et al. Risk factors for 6-month continuation of S-1 adjuvant chemotherapy for gastric cancer. Gastric Cancer. 2013; 16: 133-9.

9. Shirasaka T, Shimamato $\mathrm{Y}$, Ohshimo $\mathrm{H}$, et al. Development of a novel form of an oral 5-fluorouracil derivative (S-1) directed to the potentiation of the tumor selective cytotoxicity of 5 -fluorouracil by two biochemical modulators. Anticancer Drugs. 1996; 7: 548-57.

10. Yamanaka T, Matsumoto S, Teramukai S, et al. Analysis of risk factors for severe adverse effects of oral 5-fluorouracil S-1 in patients with advanced gastric cancer. Gastric Cancer. 2007; 10: 129-34. 